\title{
The Competing Effects of Stability and Humidity on Subtropical Stratocumulus Entrainment and Cloud Evolution from a Lagrangian Perspective
}

\author{
RYAN EASTMAN AND ROBERT WOOD \\ Department of Atmospheric Sciences, University of Washington, Seattle, Washington
}

(Manuscript received 24 January 2018, in final form 9 May 2018)

\begin{abstract}
The evolution of subtropical stratocumulus clouds and the boundary layer is studied on daily time scales from the Lagrangian perspective, following the flow. Measures of humidity above the boundary layer and of inversion strength are obtained from reanalysis data, and their effects on the Lagrangian evolution of cloud cover and the boundary layer are compared. An analysis that disentangles these variables and tests their effects independently is developed. Increased inversion strength and increased humidity above the boundary layer lead to anomalously persistent cloud cover and slower Lagrangian deepening of the boundary layer. These parameters affect the stratocumulus boundary layer in different ways: inversion strength controls the buoyancy difference across the inversion, while humidity differences affect both the radiation balance and rate of cloud drop evaporation at cloud top. The relative strengths of the two effects of humidity are compared using two products: the entraining humidity in the layer directly above the inversion and the radiating humidity, which is the mean humidity in the column above the entraining humidity. Results show that the variability in the radiating humidity is the primary driver of Lagrangian boundary layer depth changes, but entraining humidity variation is mostly responsible for altering cloud lifetime.
\end{abstract}

\section{Introduction}

In the subtropics, the interactions between stratocumulus clouds (Sc), shortwave radiation, and longwave radiation combine to cause a net cooling to the climate (Chen et al. 2000; Stephens and Greenwald 1991; Hartmann et al. 1992). Given their immense sizes, variability in these cloud decks could have substantial impacts on climate worldwide, so a thorough understanding of their evolution and mechanics is essential (Randall et al. 2007; Greenwald et al. 1995). Eastman and Wood (2016) and Eastman et al. (2017) show that anomalously low overlying humidity and low lower-tropospheric stability (LTS; defined as the difference in potential temperature $\theta$ between 700 and $1000 \mathrm{hPa}$ ) act to cause anomalous deepening in the boundary layer and a shortening of cloud lifetime. Eastman et al. (2017) goes on to show that LTS anomalies (relative to a 100-day running mean) are anticorrelated with $700-\mathrm{hPa}$ humidity anomalies, creating counteracting forces on cloud evolution and the deepening rate of the boundary layer. Further complicating the picture are two key mechanisms by which overlying

\footnotetext{
Corresponding author: Ryan Eastman, rmeast@atmos.washington. edu
}

humidity can alter the boundary layer: (i) changing the radiative balance at cloud top and (ii) altering the amount of evaporative cooling at cloud top and drying within the cloud. Here, we seek to untangle the effects of stability and both effects of humidity on boundary layer cloud evolution using a Lagrangian framework.

In the subtropics off the west coasts of the major continents, a cool, humid, well-mixed boundary layer persists above the cold ocean surface and below the warm overlying troposphere. This planetary boundary layer (PBL) deepens as it advects offshore and equatorward. Stratiform clouds form in the upper portion of the PBL (Wood 2012). Clouds emit longwave radiation, which at cloud top is less compensated by downwelling radiation, causing a net radiative cooling at cloud top, sharpening the inversion between the PBL and overlying atmosphere (Lilly 1968; Nicholls 1989; Paluch and Lenschow 1991; Krueger et al. 1995). The radiative cooling at cloud top causes air parcels to become negatively buoyant. These negatively buoyant parcels then sink downward, driving a turbulent overturning (Lock and Macvean 1999b). Cloud-top cooling is more pronounced at night when downwelling shortwave radiation is not present, leading to deeper and more turbulent boundary layers at night. 
In the zone between the turbulent cloud top and the overlying warm and dry air, dry air tends to entrain downward and mix into the PBL, deepening, warming, and drying the cloudy boundary layer (Mellado 2017; Yamaguchi and Randall 2012). The evaporation of cloud droplets into the entraining dry air at cloud top further exacerbates the cloud-top cooling, driving more turbulence (Siems and Bretherton 1992; Mellado et al. 2009; Lock and Macvean 1999a). The rate at which the air at cloud top entrains into the PBL is controlled by the buoyancy differences across the inversion, the humidity of the entrained air, and the strength of the turbulence at cloud top. Entrainment can also be enhanced by a warming sea surface below, which can impart more energy into the PBL through latent heating (Wyant et al. 1997). Entrained warm, dry air also raises the lifted condensation level, further thinning the cloud from below. The stratocumulus-topped PBL eventually deepens and dries while cumulus clouds begin to form below the stratiform layer, which modify the moisture and aerosols rising into the Sc. The overlying Sc eventually break up and give way to the trade cumulus clouds as they continue to advect equatorward and offshore.

Increased humidity overlying the PBL slows the rate of Lagrangian PBL deepening (Eastman et al. 2017) by thwarting cloud-top cooling through two different mechanisms. Greater humidity at cloud top leads to less evaporation of cloud droplets before the cloud top reaches saturation, causing less cooling and less entrainment. Additionally, a more humid troposphere above the PBL will produce an increase in downwelling longwave radiation, offsetting the energy loss to upwelling radiation, reducing cooling, turbulence, and entrainment. This effect was demonstrated by Christensen et al. (2013): Increased downwelling longwave radiation from high clouds was associated with shallower nighttime Sc cloud-top heights.

Cloud amount is affected by humidity through the above-mentioned mechanisms. Bretherton et al. (2013) present modeling results showing how Sc decks may respond to future climate scenarios where climate models predict changes in the free-tropospheric temperature and humidity above our study regions. Bretherton et al. (2013) show a radiative response to increased emissivity above the PBL predicting thinning, lowering Sc because of a reduction in cloud-top cooling and a weakening of the associated turbulence. Decreased free-tropospheric relative humidity in the subtropics (our region of study) will drive entrainment of drier air, leading to evaporating and further thinning Sc. Finally, a warmer free troposphere relative to the sea surface may lead to stronger buoyancy gradients across the inversion, limiting entrainment of drier air and prolonging Sc lifetime.
The virtual potential temperature (and therefore buoyancy) gradients across the inversion stem primarily from differences in temperature, though also humidity, between the PBL and the overlying lower troposphere. There are several ways to quantify these temperature and humidity gradients. Variability of the temperature difference above and below the inversion is quantified by the LTS, defined as the difference in potential temperatures between air at the surface and air above the inversion, usually $700 \mathrm{hPa}$ (Klein and Hartmann 1993). Wood and Bretherton (2006) quantified another stability parameter that includes LTS but also accounts for the often meantemperature-dependent free-tropospheric lapse rate in their calculation of the estimated inversion strength (EIS). In the subtropics, the free-tropospheric temperature shows little variability, so EIS and LTS should behave similarly there. Additionally, there are two quantities that account for the effects of both temperature and humidity gradients across the inversion: the cloud-top entrainment instability (CTEI) parameter $\kappa$ (Deardorff 1980; Randall 1980) and the estimated cloud-top entrainment index (ECTEI; Kawai et al. 2017). The estimated cloud-top entrainment index and $\kappa$ account for temperature and humidity differences, $\kappa$ by comparing values directly above and below the inversion and ECTEI by comparing values between the surface and $700 \mathrm{hPa}$. One limitation of the $\kappa$ parameter as a predictor variable for cloud change is that it assumes that the cloudy air has an unlimited supply of liquid water content for the evaporative cooling of the entraining air.

While $\kappa$ and ECTEI do account for the role that entraining humidity plays in modulating cloud droplet evaporation, the radiative effects of the overlying humidity are not accounted for by these parameters nor by LTS and EIS. The fraction of variability of cloud-top mixing, and therefore entrainment, caused by cloud drop evaporation versus radiative cooling is an area of ongoing research. Cloud-top mixing was shown by Yamaguchi and Randall (2012) using a large-eddy simulation to be the dominant cooling process for air parcels entraining into Sc. They go on to show that radiative cooling maintains the boundary layer turbulence. This question has been further explored by many other modeling studies utilizing "smoke clouds," where clouds are permitted to emit longwave radiation but cloud drops do not evaporate (Lilly 1968; de Lozar and Mellado 2015; Moeng et al. 1999; Stevens and Bretherton 1999; de Lozar and Mellado 2013; Lock and Macvean 1999b). Smoke clouds can be compared to water clouds to see how they evolve when only radiative processes are occurring. Though both effects are shown to be important, there is little certainty about which effect dominates, and no observational studies have been able to verify these results. 
Further complicating the observational study of parameters driving entrainment is the common cooccurrence of opposite-sign humidity and stability variation seen in Eastman et al. (2017, their Tables 1 and 2). That work shows that when humidity at $700 \mathrm{hPa}$ is anomalously high, LTS is often anomalously low, and vice versa. The observed anticorrelation may be due to meteorological effects or could be driven by the inversion strength. A weaker inversion allows more mixing between the humid boundary layer and drier upper troposphere (Galewsky 2018). Regardless of the cause, this anticorrelation means that the true effects of low LTS (forcing more entrainment, PBL deepening, and Sc breakup) are masked by the competing effects of the collocated high humidity above the PBL (forcing less entrainment, PBL shallowing, and Sc persistence).

In this work, we expand on the work of Eastman et al. (2017) to show how humidity and stability individually act on entrainment and cloud cover evolution in the PBL and to isolate and compare the differing effects of humidity. This work uses a Lagrangian framework to track individual cloud scenes as they advect offshore and deepen while interacting with the overlying atmosphere. Cloud amount and the depth of the PBL are measured using A-Train satellites every $12 \mathrm{~h}$ for every parcel. The effects on entrainment and cloud evolution of four different quantifications of stability (LTS, EIS, $\kappa$, and ECTEI) are compared, and confounding humidity anomalies are addressed. The effects of two humidity products derived from the European Centre for Medium-Range Weather Forecasts (ECMWF) interim reanalysis (ERA-Interim) are compared: the entraining humidity, which is the layer directly above the inversion, and the radiating humidity, defined as the column-mean humidity overlying the entraining humidity, but below $700 \mathrm{hPa}$. Using these products and the Lagrangian framework, we will assess whether the radiative or evaporative effects of the overlying humidity dominate the entrainment rate and drive cloud changes in the stratocumulus-topped PBL.

\section{Data}

\section{a. Winds from the ERA-Interim, sampling trajectories}

This Lagrangian study uses samples of cloud cover and PBL depth taken along trajectories in four subtropical ocean basins, the northeast Pacific, southeast Pacific, southeast Atlantic, and east Indian oceans, using the same set of trajectories as Eastman et al. (2017). Winds driving these trajectories are sourced from the ERAInterim dataset at $0.75^{\circ}$ latitude-longitude resolution
(Dee et al. 2011). We use the two-dimensional wind field at $925 \mathrm{hPa}$, assuming that this approximates the cloud level within the PBL. The vertical wind component is not considered since trajectories are required to follow the boundary layer and not the upper-level winds. Trajectories are initiated along the A-train swath to get 0 -h observations from National Aeronautics and Space Administration (NASA) satellites CloudSat and Cloud-Aerosol Lidar and Infrared Pathfinder Satellite Observations (CALIPSO) and run forward for $48 \mathrm{~h}$. This study uses 169824 individual trajectories calculated for the years 2007-10. Trajectories observed to travel in an onshore direction rather than offshore are removed from the analysis. Observations taken over land are also removed. Examples of these trajectories in each of our four study regions are shown in Fig. 1, where subsets (1 in 200) of our trajectories are plotted in each region.

Trajectories are sampled every $12 \mathrm{~h}$ with every A-Train satellite overpass at approximately 0130 and 1330 local time. At each sampling point, we sample a 100-km radius. All ERA-Interim data products sampled are interpolated to match the Moderate Resolution Imagine Spectroradiometer (MODIS level-3 (L3) $1^{\circ} \times 1^{\circ}$ latitude-longitude grid. If a gridbox center (at the $0.5^{\circ}$ point) falls within the sampling radius, then that entire box is sampled. The mean of all grid boxes within the sampling radius is then calculated for each sample.

\section{b. Cloud cover from MODIS}

Cloud cover data observed during the day and night come from the MODIS Collection 6 cloud-mask product (Hubanks et al. 2008; Oreopoulos 2005), identical to the cloud product used in Eastman et al. (2017). The MODIS sensor samples a swath that is over $2000 \mathrm{~km}$ wide, which allows for nearly global sampling each day. However, clouds seen at the edge of the swath are sampled at a much wider angle than at nadir. This causes a sampling bias because the sensor's pathlength through horizontally developed clouds is longer at wider angles and the edges of vertically developed clouds fill more of the sampled pixel. This causes an overestimation of cloud amount at high sensor zenith angles, detailed in Maddux et al. (2010). This overestimation can bias our cloud cover results since all 0-h trajectory samples are taken at a low angle, but later samples are unconstrained. To account for this, a sensor zenith angle correction is applied to all cloud retrievals, as described and applied in Eastman and Wood (2016). Briefly, that correction is based on a plot of mean cloud cover as a function of sensor viewing angle bins with the cloud cover at nadir subtracted from each value. A polynomial is fit to the plot, which provides a mean zenith angle bias 
b) SE Pacific

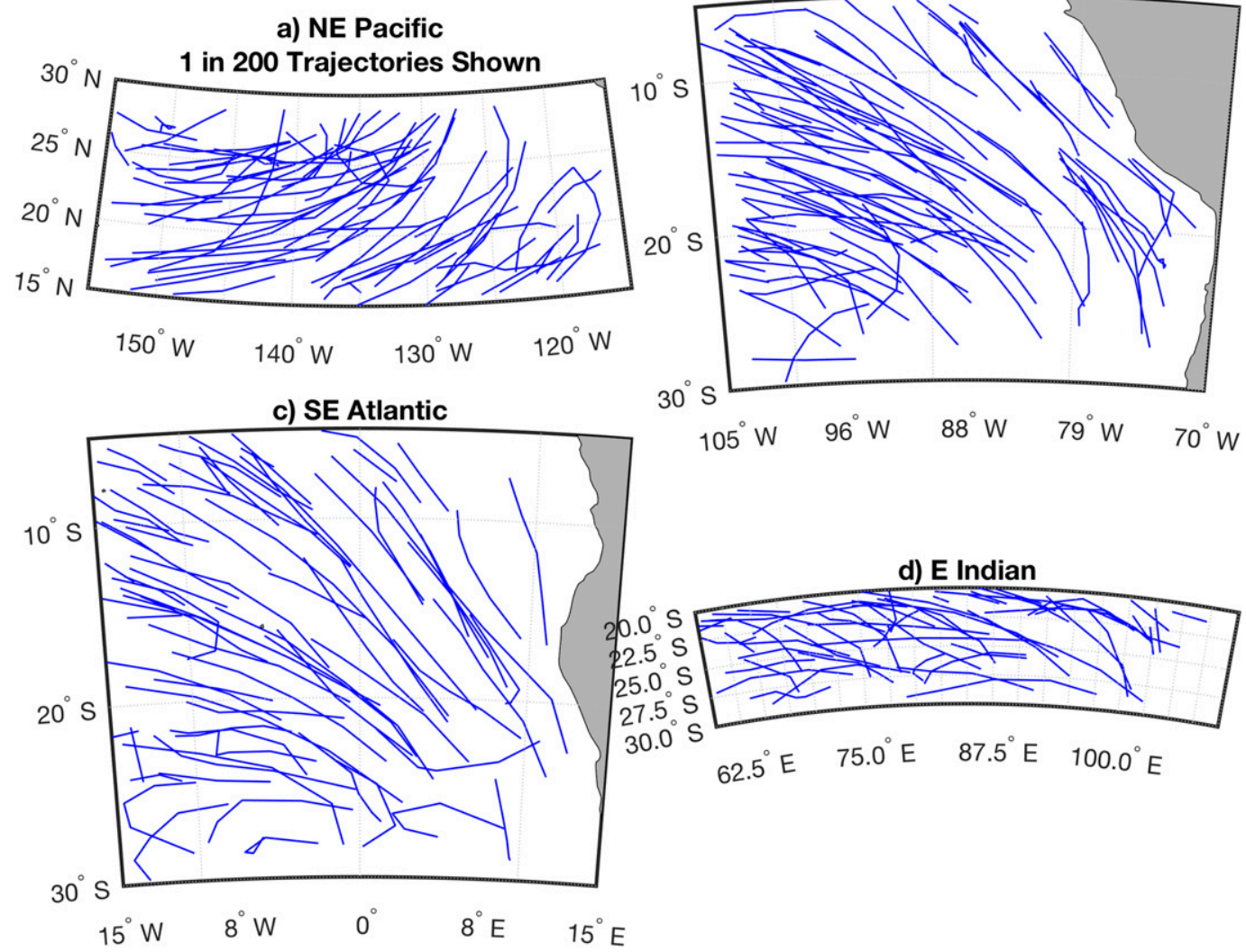

FIG. 1. A subset ( 1 in 200) of the $\sim 170000$ trajectories within the four regions of study. Trajectories run for $48 \mathrm{~h}$ and are calculated using the $u$ (zonal) and $v$ (meridional) wind fields at $925 \mathrm{hPa}$ from the ERA-Interim.

for any given sensor viewing angle. That bias is then subtracted from each MODIS cloud amount.

\section{c. PBL depth from MODIS and ERA-Interim}

The depth of the PBL is estimated from the difference in temperature between the boundary layer cloud tops and the sea surface. The sea surface temperature (SST) is assumed to match the surface skin temperature from the ERA-Interim. The temperatures of the PBL cloud tops are taken from a routine using collocated observations between the MODIS Collection 6 cloud-top temperature (CTT) joint histograms (King et al. 2003) and the vertical feature mask (VFM; Vaughan et al. 2004) product from CALIPSO. Details about this routine are available in Eastman et al. (2016, 2017). Briefly, the PBL top seen by CALIPSO is based on a frequency distribution of cloud-top heights over a 200-km-wide sample. These frequency distributions can contain multiple peaks in cases where cloud decks are multilayered. The PBL depth is assigned to the highestaltitude peak in the frequency distribution. These CALIPSO PBL measurements are then compared to collocated MODIS cloud-top temperature histograms within $1^{\circ} \times 1^{\circ}$ grid boxes. Representative cloud-top temperature bins are then determined based on cloud amount and the collocated CALIPSO measurement, and this is applied to all histograms throughout the MODIS swath.

\section{d. Entraining and radiating humidity products}

All reanalysis humidity values are taken from the specific humidity fields contained in the ERA-Interim dataset. Two unique humidity products are generated here using these fields: the "radiating humidity" and the "entraining humidity." The entraining humidity is defined as the specific humidity in the layer directly above the inversion as determined by the ERA-Interim temperature profile. We define the inversion as the layer showing the largest rate of potential temperature increase with decreasing pressure (maximum $\Delta \theta / \Delta P$, where $\Delta$ describes the change with height). The radiating humidity is the mean humidity in the column above the entraining humidity and below $700 \mathrm{hPa}$. This is shown more clearly in Fig. 2 alongside the vertical profile of potential temperature. All ERA-Interim pressure levels used are plotted in Fig. 2. We choose the 700-hPa 


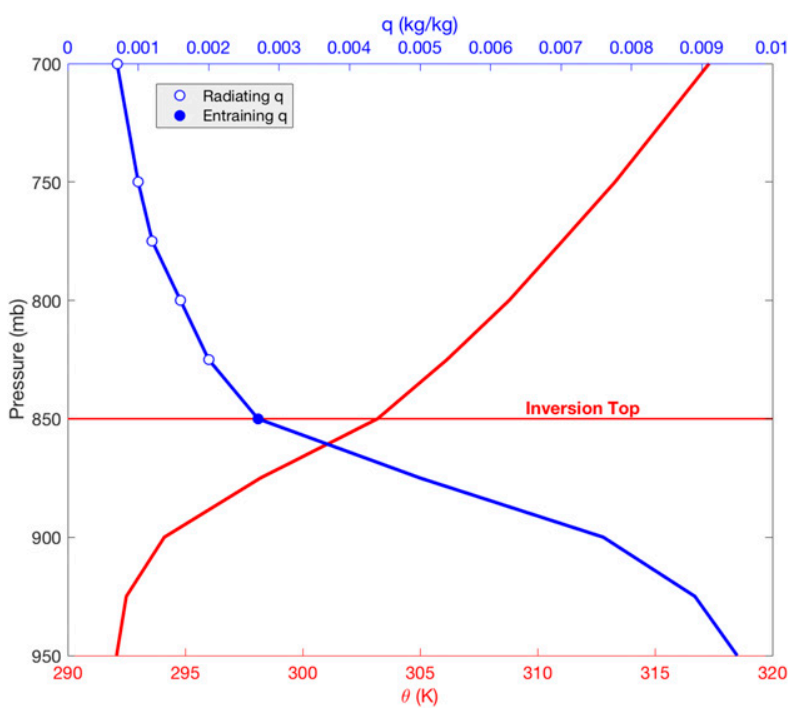

FIG. 2. An idealized vertical profile of potential temperature $\theta$ (thick red line), with the inversion top shown as the thin red line. The inversion layer is assumed to be the layer showing the largest increase in $\theta$ with height. An idealized vertical profile of specific humidity (blue line). The solid dot represents the entraining humidity. The hollow dots represent the radiating humidities, which are averaged in our calculation of radiating humidity.

cutoff since the air temperature at higher levels may not be warm enough to have a significant radiative effect. Scenes with interfering high clouds are removed from the analysis.

To verify that the radiating humidity is in fact radiating, we have compared our values of radiating humidity with radiative fluxes from the CloudSat level-2B fluxes and heating rate (FLXHR) lidar dataset (Henderson et al. 2013). The FLXHR lidar dataset uses temperature and moisture profiles from the ECMWF analyses along with cloud and aerosol profiles obtained from CloudSat and CALIPSO to estimate upwelling and downwelling radiative fluxes. Fluxes are calculated for shortwave and longwave radiation for $\sim 240$-m vertical layers along the CloudSat curtain. To compare with our radiating humidity data, we obtain both the downwelling flux and flux divergence (upwelling minus downwelling) of longwave radiation at the cloud-top level. The flux divergence measures the net amount of longwave radiation lost to space at cloud top, providing a measure of cloud-top cooling. Calculations are done at each 200-km-wide, 0-h sampling point for the first 100 days of 2007 , creating $\sim 8500$ individual comparisons. The correlation between the radiating humidity and the downwelling longwave flux at cloud top is $r=0.62$. The correlation between the radiating humidity and the flux divergence at cloud top is $r=-0.48$. Both correlation coefficients are significant at the $99 \%$ level, and the correlations show that our radiating humidity product is significantly and positively associated with downwelling radiation and significantly negatively associated with cloud-top cooling.

\section{e. Stability parameters from ERA-Interim}

Several different measures of inversion strength are compared and calculated using fields from the ERAInterim. The lower-tropospheric stability is defined here as the difference in potential temperature between 700 and $1000 \mathrm{hPa}$ :

$$
\mathrm{LTS}=\theta_{700}-\theta_{1000} .
$$

The EIS is calculated as shown in Wood and Bretherton (2006):

$$
\mathrm{EIS}=\mathrm{LTS}-\Gamma_{m}^{850}\left(z_{700}-\mathrm{LCL}\right) .
$$

The EIS uses LTS but subtracts the moist adiabatic potential temperature gradient $\Gamma_{m}$ scaled by the difference between the height of the 700-hPa surface and lifting condensation level (LCL), defined in Eq. (2) here and in Eq. (5) of Wood and Bretherton (2006). The parameter $z_{700}$ is the height of the $700-\mathrm{hPa}$ surface. The LCL is approximated as in Eq. (24) of Lawrence (2005). The EIS parameter was formulated as a single measure of inversion strength that could explain boundary layer cloud variability in the tropics, subtropics, and midlatitudes. The ECTEI (Kawai et al. 2017) uses the EIS and also includes the evaporative effects of the humidity gradient between the surface and $700 \mathrm{hPa}$ :

$$
\mathrm{ECTEI}=\mathrm{EIS}-\beta\left(L_{v} / c_{p}\right)\left(q_{\text {surf }}-q_{700}\right),
$$

where $\beta$ is shown to be a constant equal to 0.23 [Kawai et al. 2017, their Eq. (3)], $L_{v}$ is the latent heat of vaporization, $c_{p}$ is the specific heat, and $q$ is the specific humidity at the specified level. Finally, we analyze the CTEI parameter (or $\kappa)$ :

$$
\kappa=1+\left[\Delta \theta / \Delta q\left(L_{v} / c_{p}\right)\right],
$$

where $\Delta \theta$ and $\Delta q$ are the differences in potential temperature and humidity (respectively) across the inversion as seen by the ERA-Interim and described in section $2 \mathrm{~d}$. The $\kappa$ parameter differs from the other three by comparing values directly above and below the inversion rather than between the surface and $700 \mathrm{hPa}$.

\section{f. Column-integrated water vapor from AMSR-E}

The aforementioned measures of free-tropospheric humidity (section $2 \mathrm{~d}$ ) are all based on reanalysis products. An independent measure of humidity is provided by the column-integrated water vapor (CIWV) sampled 
by the Advanced Microwave Scanning Radiometer for EOS (AMSR-E; Wentz and Meissner 2004), using the 19-, 23-, and 37-GHz microwave channels.

\section{g. Calculating anomalies}

In much of this work, we compare anomalies of our variables instead of the actual values. This is to ensure that differences in trajectory length and differing geographical start and end points do not bias any comparisons. To calculate an anomaly, we subtract the 100-day running mean from each $1^{\circ} \times 1^{\circ} \mathrm{L} 3$ grid box centered on each day. Anomalies are calculated individually for day and night to avoid any biases related to the diurnal cycle. This is done identically to Eastman et al. (2017). Anomalies are denoted using a prime symbol.

\section{Methods and results}

a. Quantifying and comparing cloud and boundary layer evolution in a Lagrangian framework and the cloud cover response to LTS

The goal of this work is to compare changes over time in cloud and boundary layer properties following the flow in the PBL. We compare changes in groups of trajectories selected by their initial conditions. When grouping trajectories by environmental variables (i.e., comparing trajectory groups with above- or below-average starting LTS), it is common that the frequency distributions of 0 -h cloud and boundary layer properties will differ between groups. These differences in initial distributions can drive Lagrangian changes independent of the environmental variables being tested, as shown below. Eastman and Wood (2016) showed that this can be accounted for in several ways, one of which is applied here.

An example of this process is shown in Figs. 3-5. Figure 3 shows the 24-h Lagrangian changes in cloud cover anomalies ( $\triangle \mathrm{CCA}$, where $\Delta$ describes the change in time) as a function of their 0 -h values. A linear relationship is apparent, shown by the red line fit to the plot using a robust linear regression. This figure demonstrates that 24-h changes in cloud cover on average tend to act to compensate initial anomalies. A trajectory with anomalously low cloud cover at $0 \mathrm{~h}$ will most likely see a subsequent increase in cloud cover, and vice versa.

In Fig. 4, we show frequency distributions of 0 -h cloud cover anomalies for two groups of trajectories. Trajectories are separated by their 0 -h LTS', so we can compare the effects of LTS on cloud evolution. The blue group contains all trajectories with at- or below-zero 0-h LTS anomalies, while the red group contains all trajectories with above-zero 0 -h LTS anomalies. The red (high 0-h LTS') group shows a greater number of above-zero

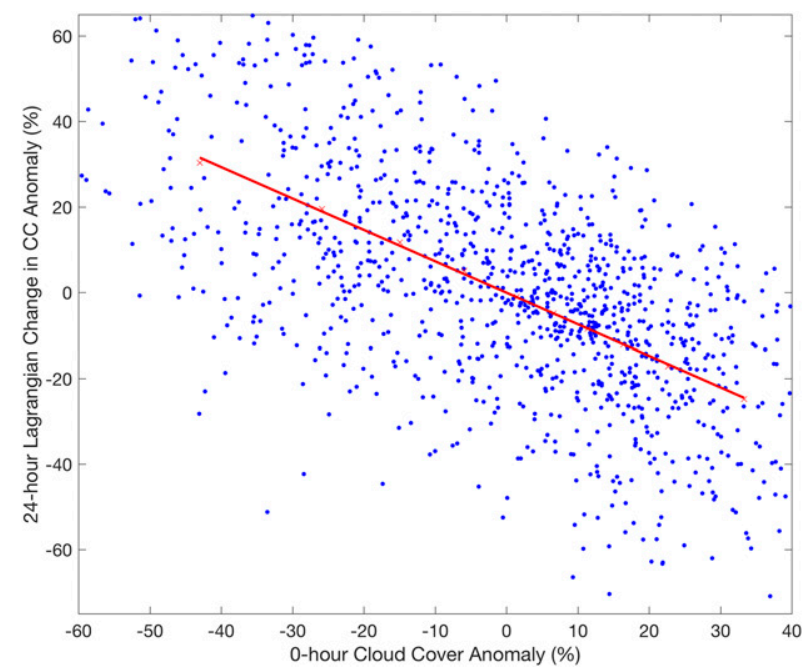

FIG. 3. Cloud cover anomalies calculated at trajectory beginning ( $0 \mathrm{~h} ; x$ axis). The subsequent 24 -h Lagrangian change in cloud cover anomaly for each trajectory ( $y$ axis). Anomalies are calculated by subtracting the 100-day running-mean cloud amount centered on each day. A linear fit calculated using a robust linear regression is also shown (red line).

cloud cover anomalies, while the blue group (low 0-h

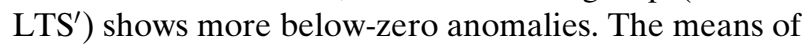
both distributions are also shown to highlight the differences in initial cloud anomalies. Because of the relationship seen in Fig. 3, the observed Lagrangian changes in cloud cover may be forced by the LTS and by the differing initial distributions shown in Fig. 4.

The Lagrangian evolutions of both groups of trajectories are shown in Fig. 5. Each group is compared to a "matched distribution," which is a group of trajectories that is randomly chosen from our entire set but forced to have an identical 0 -h frequency distribution of cloud cover anomalies. The matched distribution shows how that frequency distribution would normally evolve. By subtracting the mean cloud cover anomaly observed by the matched distribution trajectories from the anomaly observed by the trajectories grouped by 0 -h LTS, we can calculate a "residual" change. The residual is the portion of cloud cover change driven by the LTS and not by the initial distribution of cloud cover anomalies. A comparison of the residuals at $24 \mathrm{~h}$ shows that high LTS is associated with greater-than-expected cloud longevity, while low LTS is associated with less-thanexpected cloud longevity. The next several sections of this work will focus on the comparison of these residuals.

\section{b. Calculating residuals in standard deviation bins and the PBL response to EIS and LTS variability}

To compare Lagrangian changes forced by different environmental variables, we must create an equivalent 


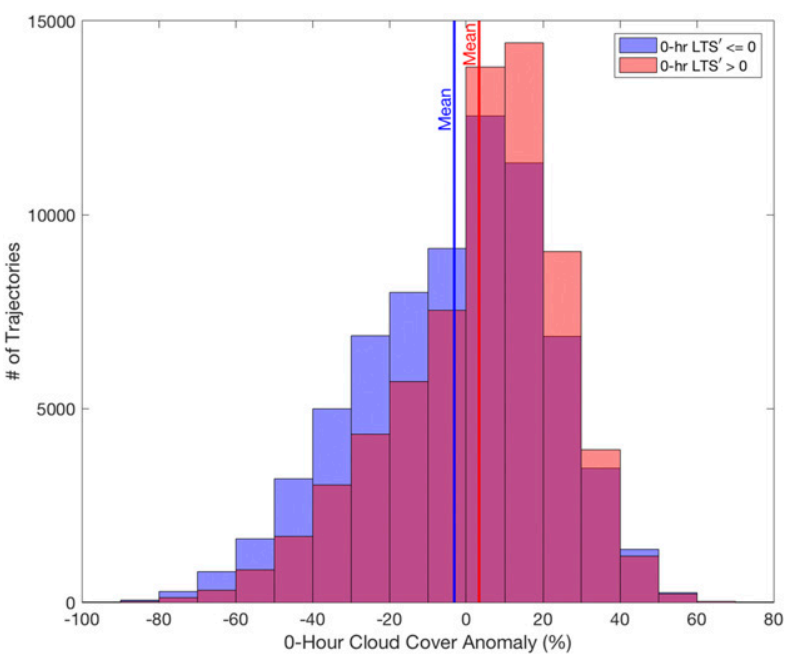

FIG. 4. Frequency distributions of cloud amount at trajectory beginnings $(0 \mathrm{~h})$ for two groups of trajectories: the blue group has below-zero LTS anomalies at $0 \mathrm{~h}$, and the red group has above-zero LTS anomalies at $0 \mathrm{~h}$. The mean 0 - $\mathrm{h}$ LTS anomaly for each group is shown as a vertical line of the corresponding color.

measure of variability between those variables. We do this using the standard deviations $\sigma$ of the environmental variables as in Eastman et al. (2017). So in order to compare the effects of two different measures of stability, in this case LTS and EIS anomalies, we first calculate the standard deviations of both variables. Then we divide the LTS and EIS anomalies by their respective standard deviations. Finally, we can calculate a residual change in cloud cover or PBL depth anomalies for bins of $\sigma$, in this case for $-2 \leq \sigma<-1,-1 \leq \sigma<0,0 \leq$ $\sigma<1$, and $1 \leq \sigma \leq 2$. Whichever variable shows the largest mean residual change in cloud anomaly or PBL depth anomaly for a given $\sigma$ bin is considered the more powerful driver of the Lagrangian change.

In Figs. 6 and 7, we apply the above methodology to changes in PBL depth for $\sigma$ bins of LTS (Fig. 6) and EIS (Fig. 7) anomalies. Figures 6a and 7a show a frequency distribution of the number of trajectories for a range of $\sigma$ values. The four $\sigma$ bins defined above are discernable by color. Figures $6 \mathrm{~b}$ and $7 \mathrm{~b}$ show the mean 24 -h evolution of PBL depth anomalies ( $\left.\mathrm{PBL}^{\prime}\right)$ within the $\sigma$ bins (color) and the evolution of $\mathrm{PBL}^{\prime}$ for a group of randomly chosen trajectories from a matched distribution (gray). The gray line shows smaller error bounds because it is the mean evolution from 20 matched distributions. The residual is defined as the difference in the 24-h $\triangle \mathrm{PBL}^{\prime}$ between the randomly matched trajectories and the trajectories grouped by LTS' $^{\prime}$ or EIS' (the colored line minus the gray line). The residuals are plotted per $\sigma$ bin in Figs. $6 \mathrm{c}$ and $7 \mathrm{c}$ where a linear fit is shown and the slope is calculated in meters per $\sigma$.

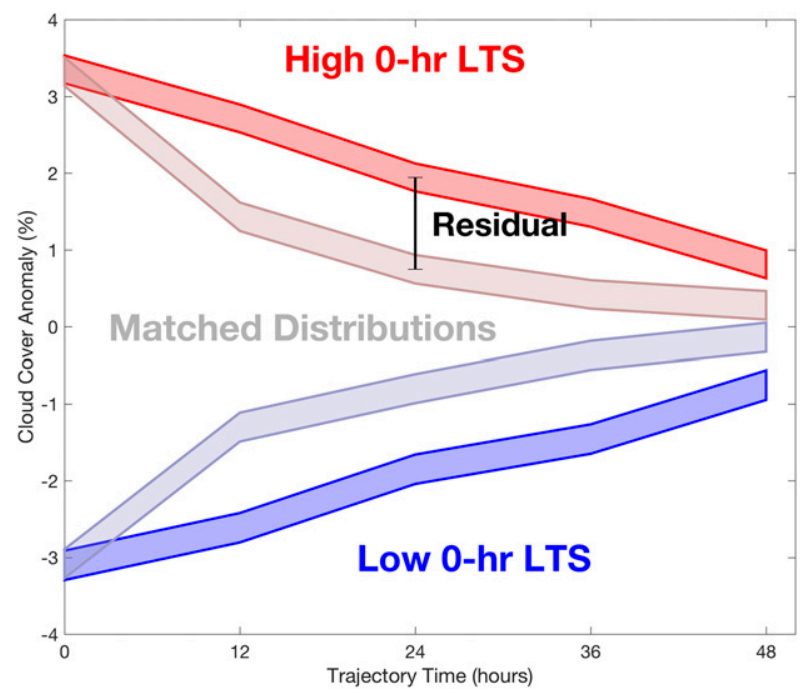

FIG. 5. The mean 48-h Lagrangian evolution of cloud cover anomalies for the two groups of trajectories shown in Fig. 4 (bright red and bright blue lines). Uncertainty is represented by line thickness and is calculated as the $2 \sigma$ standard error of the mean. Also shown in corresponding faint colors are two other sets of randomly chosen trajectories with identical frequency distributions at $0 \mathrm{~h}$ (matched distributions). The residual change in cloud cover is defined as the difference in changes in cloud cover between the two sets. The residual is the portion of cloud cover change independent of the initial frequency distribution.

Figure $6 \mathrm{c}$ shows that PBL deepening is associated with anomalously low 0-h LTS, an expected result given that low LTS is associated with less of a buoyancy difference across the inversion. However, Fig. 7c shows that PBL deepening and EIS apparently experience the opposite relationship. This is due to the correlation between EIS and the humidity above the PBL, which obscures the EIS-PBL-deepening relationship and is accounted for in the following section.

\section{c. Accounting for variable cross correlation, untangling the effects of humidity from inversion strength}

In Eastman et al. (2017), LTS anomalies (LTS') were shown to be negatively correlated with $700-\mathrm{hPa}$ specific humidity anomalies (ERA-Interim $q_{700}^{\prime}$ ). They went on to show that increased $q_{700}^{\prime}$ leads to a shallowing PBL. In Fig. 8, we test that second conclusion with satellite data using the column-integrated water vapor anomalies $\left(\mathrm{CIWV}^{\prime}\right)$ from AMSR-E in place of $q_{700}^{\prime}$. The correlation coefficient between all 0-h samples of CIWV' and $q_{700}^{\prime}$ is $r=0.8$. Figure 8 uses the same procedures as shown in Figs. 6 and 7 but for brevity only shows the equivalents of Figs. $6 \mathrm{c}$ and $7 \mathrm{c}$. Figure 8a expands the work from Eastman et al. (2017) to show that increased CIWV' leads to anomalous cloud persistence. Figure $8 \mathrm{~b}$ reiterates 

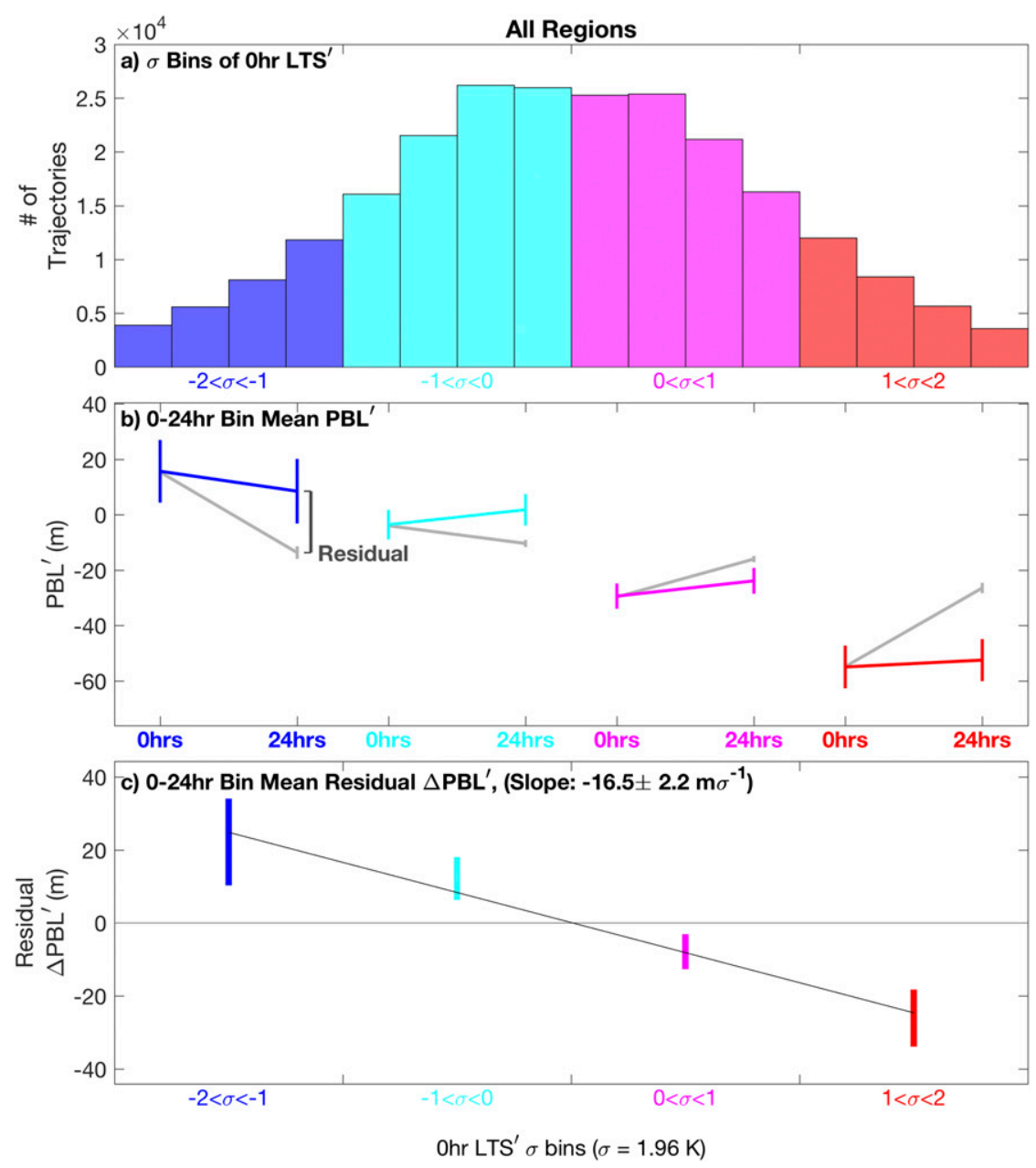

FIG. 6. (a) A frequency distribution of trajectories grouped into four bins based on their 0 -h LTS anomalies. (b) Mean and $2 \sigma$ standard error values of PBL' at 0 and $24 \mathrm{~h}$ for each of the four groups of trajectories (colored lines). Mean and standard error values for the sum of 20 sets of randomly chosen trajectories with matched 0 -h frequency distributions (gray lines). Residuals are calculated by subtracting the 24-h values of the matched trajectories from the corresponding 24-h values of the trajectories grouped by LTS' (residual = colored - gray). (c) The $0-24$-h residual $\Delta \mathrm{PBL}^{\prime}$ values for each $\sigma$ bin with $2 \sigma$ standard error ranges and a linear fit.

the established conclusions that increased $\mathrm{CIWV}^{\prime}$ forces anomalous PBL shallowing. The agreement of Fig. $8 \mathrm{~b}$ with the results of Eastman et al. (2017) taken together with the strong correlation between $q_{700}^{\prime}$ and $\mathrm{CIWV}^{\prime}$ lends a great deal of trustworthiness to the reanalysis humidity values, allowing us to continue using these reanalysis humidity fields with confidence. Correlations are calculated using coinciding 0 -h samples from each trajectory.

The relationships between $\mathrm{CIWV}^{\prime}$ and cloud and PBL evolution combined with the negative correlation between free-tropospheric humidity and LTS $(r=-0.32)$ shows that the effects of inversion strength and overlying humidity tend to offset one another. The correlation between 0-h EIS anomalies (taken at each trajectory beginning) and the corresponding $q_{700}^{\prime}$ is even more strongly negative than the correlation between LTS $^{\prime}$ and $q_{700}^{\prime}(r=-0.45$ vs $r=-0.32$ ). This stronger correlation and the results of Fig. 7 suggest that the humidity anomalies that coincide with the EIS anomalies may heavily obscure the effects on entrainment of the measure of inversion strength quantified by EIS.

To more clearly show the effect on PBL deepening of one variable independent of the other, in Eastman et al. (2017), the effect of the one variable was tested while the other was kept constant. This was done by selecting subsets of trajectories with a narrow range of one variable, while the other was unconstrained. The process 


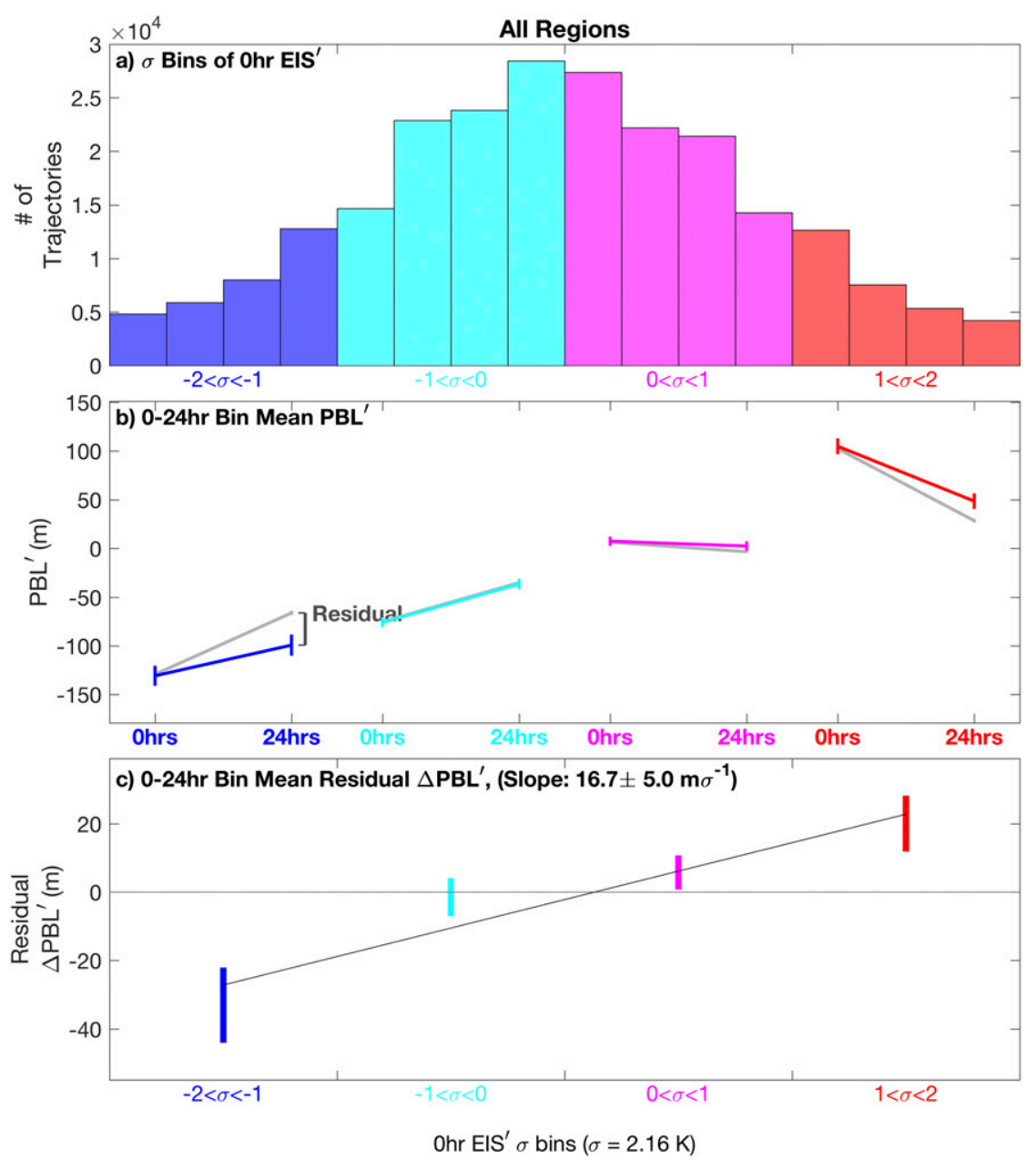

FIG. 7. As in Fig. 6, but for EIS anomalies.

from section 3b (Figs. 6, 7) was then applied to the unconstrained variable within each subset of trajectories, reducing the effects of cross correlation. We show that here in Fig. 9. In Fig. 9a, our trajectories are separated into four $\sigma$ bins; this time, the bins represent standard deviation bins of $q_{700}^{\prime}$. The analysis from Fig. 7 is then carried out for four EIS' bins within each individual $q_{700}^{\prime}$ bin. Four separate lines, one for each $q_{700}^{\prime}$ bin, are calculated as in Fig. 7c and are shown in Fig. 9b. The range of slopes defined by those four lines is listed in the subtitle and shown in Fig. 9c. There, we show that EIS does effect PBL deepening as expected but only after accounting for the confounding $q_{700}^{\prime}$.

In Table 1, we apply the above 24-h Lagrangian analysis to four stability parameters and compare their slopes for the evolution of $\mathrm{PBL}^{\prime}$ and cloud cover anomalies $\left(\mathrm{CC}^{\prime}\right)$. In addition to $\mathrm{LTS}^{\prime}$ and $\mathrm{EIS}^{\prime}$, we compare two other variables that take into account the humidity above the PBL: the ECTEI and $\kappa$. For EIS and $\mathrm{LTS}^{\prime}$, we compare slopes per $\sigma$ bin with and without accounting for $q_{700}^{\prime}$. Results show that LTS' $^{\prime}$ and EIS' display the expected negative relationships with PBL deepening after accounting for the confounding effects of $q_{700}^{\prime}$. Both $\kappa^{\prime}$ and ECTEI' showed a weaker relationship with Lagrangian PBL deepening, with ECTEI' showing a strong resemblance to EIS' (before holding $q$ constant). Since the upper humidity is an integral part of ECTEI' and EIS', we refrain from doing an analysis with $q_{700}^{\prime}$ held constant for those variables. For cloud cover evolution, ECTEI' shows the strongest relationship, with positive ECTEI' associated with more cloud cover. Both EIS' and LTS' show the expected positive relationship with $\mathrm{CC}^{\prime}$ evolution, with their effects again shown to be stronger after accounting for confounding $q_{700}^{\prime}$. The variable $\kappa$ appears to have the expected sign in both cases, but its relationship with both PBL and CC evolution is weaker than the other variables. This may be due to deficiencies in the 

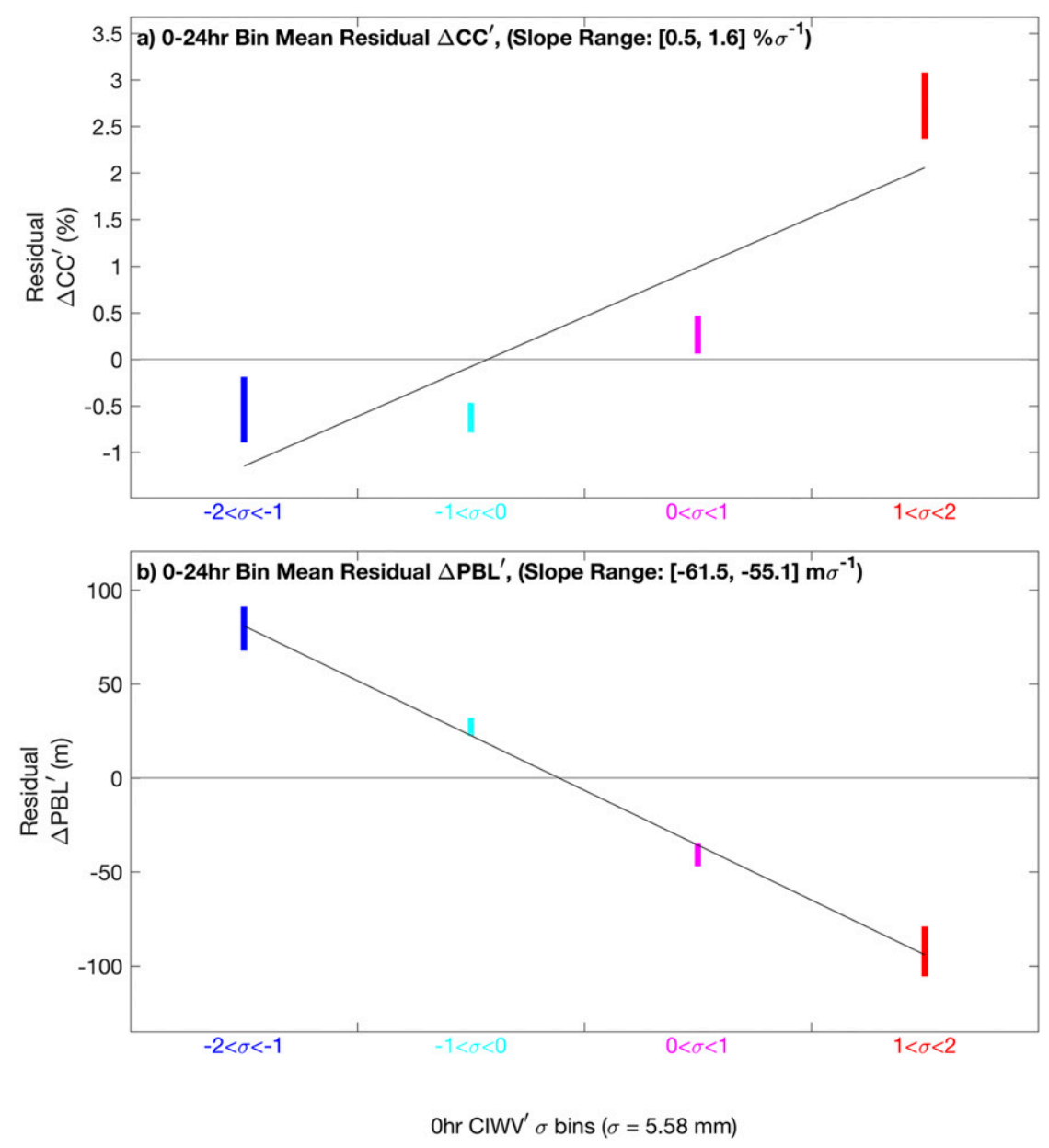

FIG. 8. (a) Residual 24-h changes in $\mathrm{CC}^{\prime}$ for $\sigma$ bins of $\mathrm{CIWV}^{\prime}$. (b) Residual 24-h changes in PBL' for $\sigma$ bins of CIWV'.

representation of the inversion in the ERA-Interim more so than any fundamental flaw in the formulation of the variable.

The differing PBL responses to $\mathrm{LTS}^{\prime}$ versus EIS' seen in Table 1 is a surprising result since both variables attempt to quantify the same thing. The major difference between EIS and LTS is that EIS accounts for variability in the free troposphere above the PBL. In doing so, EIS incorporates several additional variables that are correlated with $q_{700}$, leading to the surprising difference in PBL response compared to LTS. Specifically, EIS modifies LTS by subtracting the height difference between the LCL and the 700-hPa surface $z_{700}$, scaled by the moist-adiabatic lapse rate at $850 \mathrm{hPa} \Gamma_{m}^{\prime}$. A correlation study comparing values at our trajectory beginnings shows that ERA-Interim $q_{700}^{\prime}$ correlates negatively with the $\mathrm{LCL}^{\prime}(r=-0.38)$ and positively with $z_{700}^{\prime}$ and $\Gamma_{m}^{\prime}(r=0.17$ and $r=0.27$, respectively). Taken alongside Eq. (2), this means that these three variables all vary in concert with $q_{700}$, each individually enhancing the negative relationship between $q_{700}^{\prime}$ and EIS'.

\section{d. Assessing the effects of humidity above the PBL on entrainment and cloud evolution}

While both ECTEI and $\kappa$ do account for overlying humidity, a drawback is that they are conceived only to account for the entraining effects of humidity but do not account for the radiative impacts of the overlying humidity. This has the possible effect of underrepresenting the role played by humidity in entrainment and may be why these variables appear weaker than expected when predicting PBL deepening. Here, we will independently test the effects of the radiating and entraining humidity using the process described in Figs. 6 and 7 and the humidity quantities described in section $2 \mathrm{~d}$.

In Figs. 10 and 11, the effects of radiating and entraining humidity are compared first for bins of constant LTS $^{\prime}$, then within bins of the other humidity 

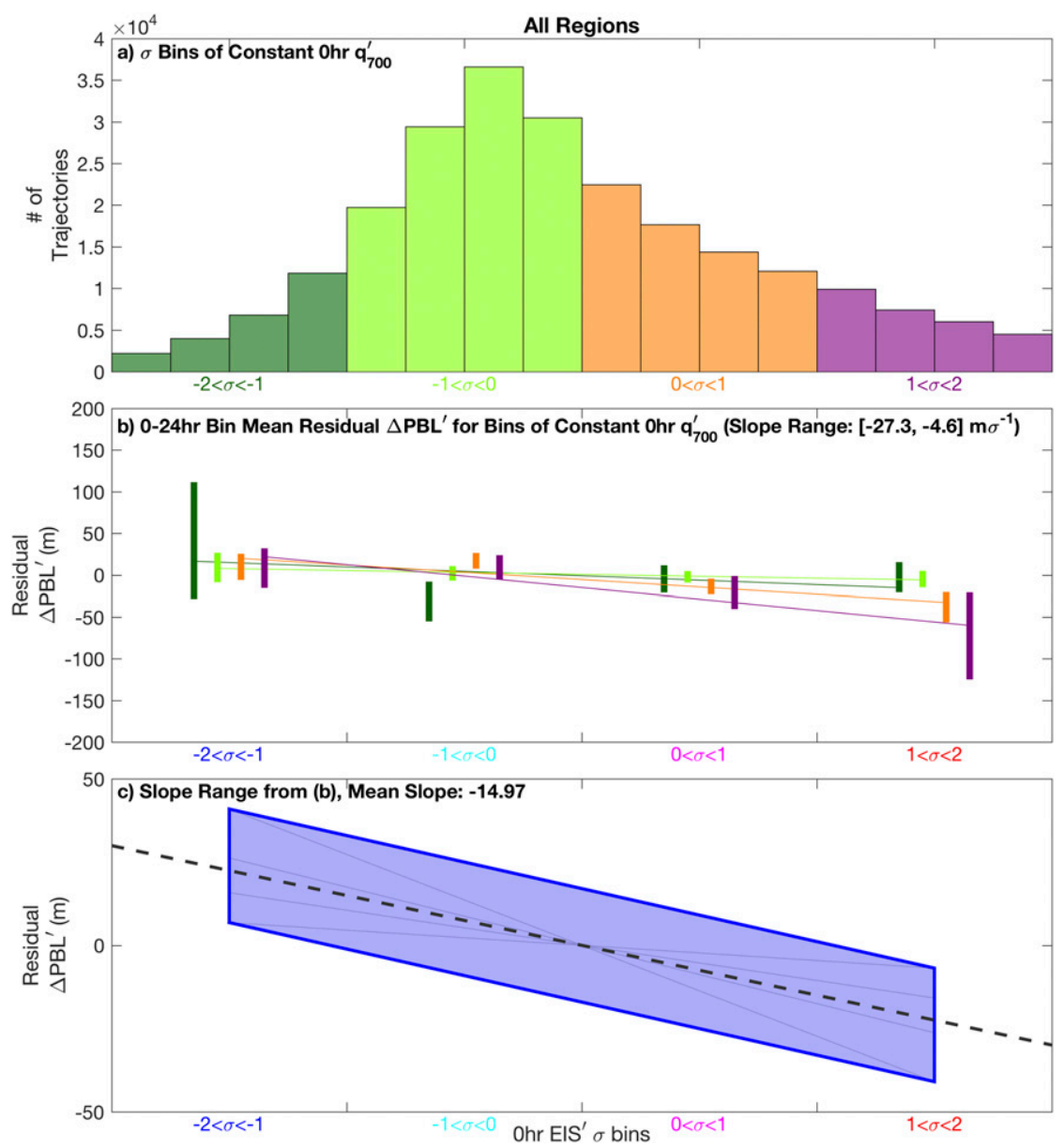

FIG. 9. (a) A frequency distribution of trajectories grouped into four bins based on the standard deviation $\sigma$ of their 0 -h $q_{700}^{\prime}$ anomalies. (b) The analysis from Fig. 7 carried out four times, once in each $q_{700}^{\prime} \sigma$ bin, creating a range of slopes while controlling for $q_{700}^{\prime}$. (c) The range of slopes shown in (b) with the mean slope shown as a dashed line and the range of uncertainty shown by the blue bounds.

product held constant in order to separate their effects. In Fig. 10, the Lagrangian changes in $\mathrm{PBL}^{\prime}$ are compared. For bins of constant LTS', the effects of the radiating humidity seen in Fig. 10b appear stronger than the effects of the entraining humidity in Fig. 10a. This is reinforced in Figs. 10c and 10d, where the effects of the radiating humidity with constant entraining humidity are stronger than the effects of entraining humidity with constant radiating humidity. A comparison of the slopes indicates that the radiating humidity is likely the

TABLE 1. The mean and range of slopes describing the change in PBL depth and cloud cover per standard deviation $(\sigma)$ bin of the listed variable. Ranges represent the mean slope plus and minus the standard deviation in the slope as shown in Fig. 8. A second mean and range of slopes is shown for the variables LTS ${ }^{\prime}$ and EIS ${ }^{\prime}$ where $700-\mathrm{hPa}$ specific humidity $q_{700}$ is held constant in bins. Prime symbols indicate anomalies. Ranges here represent the largest and smallest slopes from all four constant $q_{700}$ bins, as in Fig. 9.

\begin{tabular}{lcccc}
\hline & \multicolumn{2}{c}{$\mathrm{PBL}^{\prime}$ mean slope and range per $\sigma$ bin $\left(\mathrm{m} \sigma^{-1}\right)$} & & $\mathrm{CC}^{\prime}$ mean slope and range per $\sigma$ bin $\left(\% \sigma^{-1}\right)$ \\
\cline { 2 - 3 } & & $q_{700}$ constant & & $q_{700}$ constant \\
\hline LTS & $-16.2[-17.8,-14.7]$ & $-37[-43.3,-32.8]$ & $1.1[0.6,1.5]$ & $1.5[0.8,1.8]$ \\
EIS & $16.4[10.8,23.1]$ & $-14.9[-27.3,-4.6]$ & $0.3[-0.2,0.7]$ & $0.9[0.4,1.4]$ \\
ECTEI & $13.6[11.2,16.5]$ & & $1.6[1.3,1.8]$ & $-0.5[-0.6,-0.4]$ \\
$\kappa$ & $8.1[2.9,14.4]$ & &
\end{tabular}




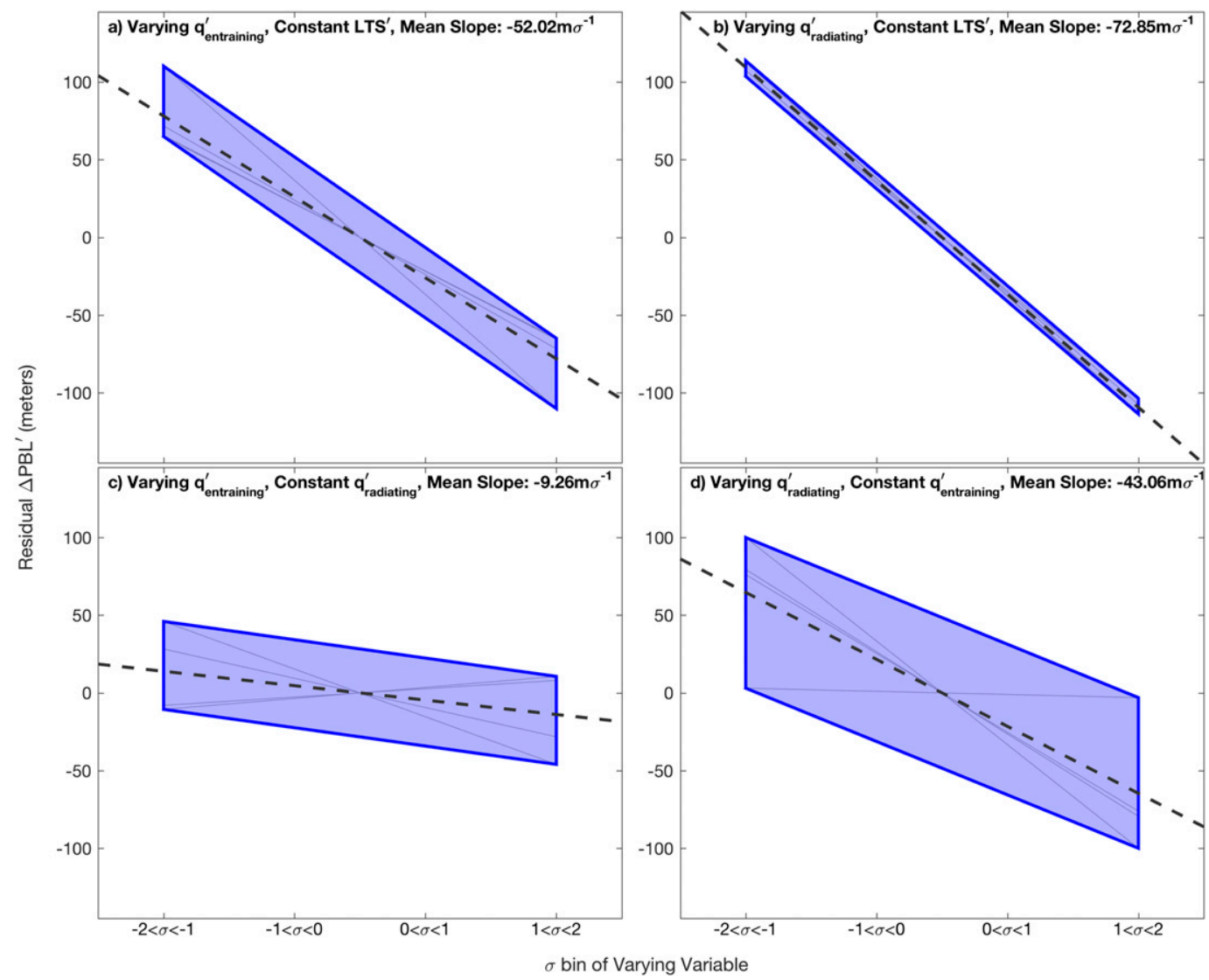

FIG. 10. Ranges of slopes for 24-h residual change in $\Delta \mathrm{PBL}^{\prime}$ for $\sigma$ bins of one varying variable while controlling for another by holding it constant, produced using the method in Fig. 8. (a) Residual $\Delta \mathrm{PBL}^{\prime}$ for $\sigma$ bins of entraining humidity $q_{\text {entraining }}^{\prime}$ while holding LTS' constant. (b) Residual $\Delta \mathrm{PBL}^{\prime}$ for $\sigma$ bins of radiating humidity $q_{\text {radiating }}^{\prime}$ while holding LTS ${ }^{\prime}$ constant. (c) Residual $\Delta \mathrm{PBL}^{\prime}$ for $\sigma$ bins of $q_{\text {entraining }}^{\prime}$ while holding $q_{\text {radiating }}^{\prime}$ constant. (d) Residual $\Delta \mathrm{PBL}^{\prime}$ for $\sigma$ bins of $q_{\text {radiating }}^{\prime}$ while holding $q_{\text {entraining }}^{\prime}$ constant.

dominant factor driving PBL deepening, and not the entraining humidity, possibly by more than a factor of 3 .

A different picture is seen in Fig. 11 when the Lagrangian evolutions of $\mathrm{CC}^{\prime}$ are compared. In Figs. 11a and $11 \mathrm{~b}$, the entraining humidity appears to be a marginally stronger driver of $\mathrm{CC}^{\prime}$ evolution compared to the radiating humidity when LTS $^{\prime}$ is held constant. In Figs. 11c and 11d, this result is greatly enhanced. The radiating humidity appears ineffective when the entraining humidity is controlled for, but the entraining humidity appears to have a strong effect when the radiating humidity is controlled for. According to this figure, the radiative effects of humidity are mostly inconsequential on cloud cover evolution despite affecting PBL growth significantly, while the entraining effects appear to be very important.

Wide error bounds in Figs. 10c, 10d, 11c, and 11d suggest that the effects of one of the two variables may be dependent on the state of the other. In Figs. 12 and 13 , we further explore this by separating our trajectories into four groups. Mean values of radiating and entraining humidity are calculated for each trajectory by averaging retrievals at all five sampling times. Four trajectory groups are chosen by combining all possible sets of trajectories with above- or below-mean radiating and entraining humidities. Results in Fig. 12 appear consistent with Fig. 10. The two sets of trajectories with aboveaverage radiating humidity are on average deeper, and they stay deeper throughout their observed evolution. The entraining humidity does appear to have an effect, with drier entraining air associated with deeper PBLs, but the effect is secondary to the radiating humidity. Figure 13 also appears consistent with Fig. 11. Trajectories that entrain humid air tend to stay anomalously cloudy, while trajectories entraining dry air tend to be less cloudy and to lose cloud cover. The radiating humidity shows little significant effect as evidenced by the overlapping error bars. This analysis suggests that the wide error bounds may be more due to noise in the data than to any specific state dependence issues. 


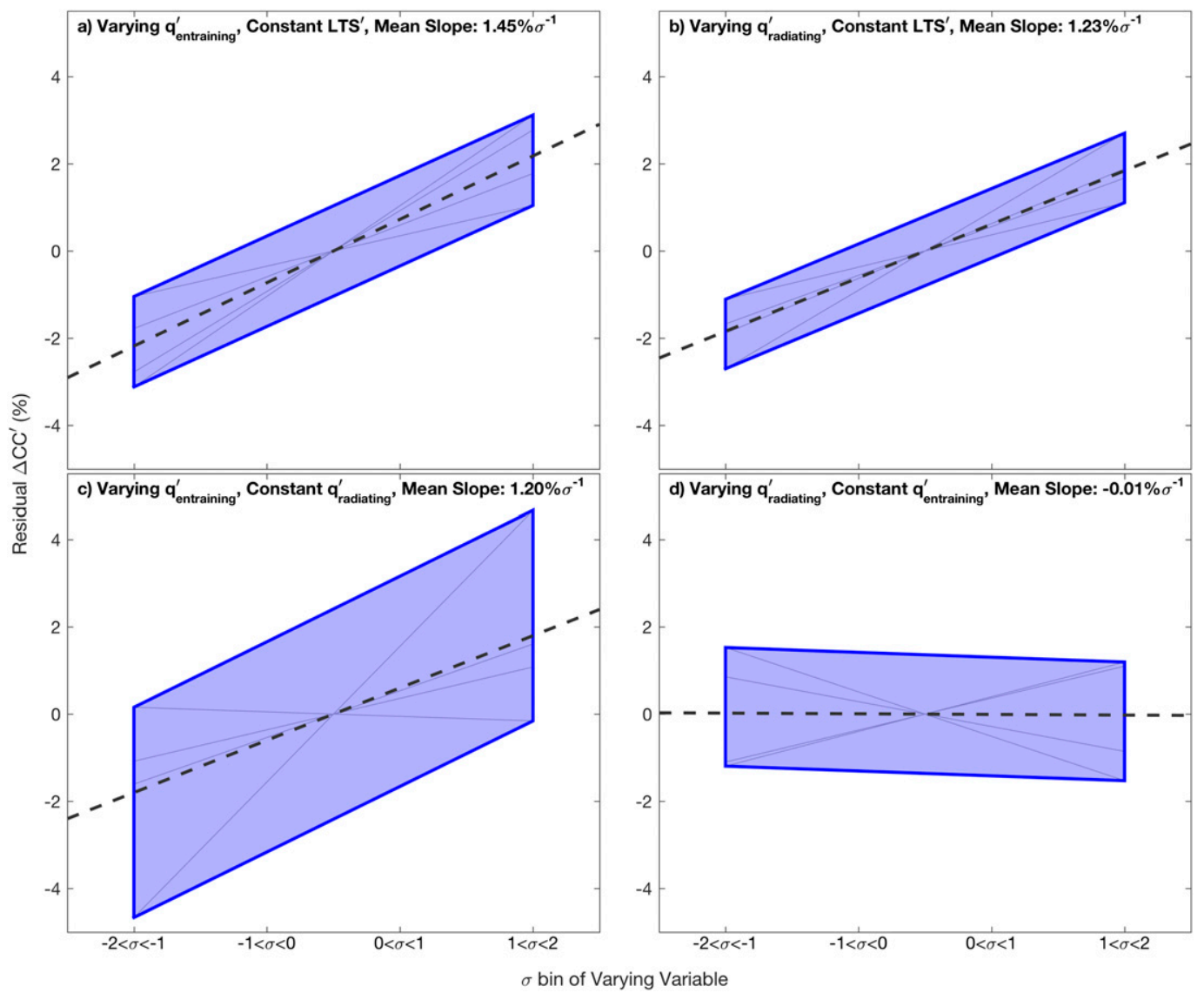

FIG. 11. As in FIG. 10, but for $\Delta \mathrm{CC}^{\prime}$.

\section{Discussion}

In Table 1 and Fig. 9, we show that LTS $^{\prime}$ and EIS' exhibit a stronger negative relationship with Lagrangian PBL deepening only after the confounding humidity is accounted for. However, adding a humidity variable to EIS', creating ECTEI', made little significant change to the relationship between EIS' or ECTEI' and Lagrangian PBL deepening. Interestingly, ECTEI' showed a much stronger positive relationship with Lagrangian cloud cover evolution when compared to EIS'. This disparity may be caused by the differing ways that overlying humidity interacts with the boundary layer and creates a need for a better variable to predict entrainment rates. Since the radiating effects of overlying humidity appear to exert more influence on PBL deepening relative to the entraining effects, a new variable would need to take into account the radiative flux divergence and associated cloud-top heating/cooling rates.

These results also stress the importance of correctly simulating both the radiating and entraining effects of humidity in climate models. Both effects are shown to significantly modify cloud cover and the PBL depth. If models are only capable of properly simulating the interaction of radiation with cloud cover but not entrainment, then the cloud response to changing humidity above the PBL is likely to be incorrect. Hopefully, this work can act as a check for the entrainment parameterizations used in current models.

Using this framework, future work may be able to address the radiative and entraining effects of not only humidity but also aerosols above the boundary layer, which often coincide with humid layers and may have comparable effects to the humidity. Also, the FLXHR lidar dataset mentioned in section 2 offers estimates of the radiative fluxes throughout the atmosphere. These estimates could be used to observationally tune and test an entrainment parameter that includes the radiative effects of the overlying humidity and aerosols. The NASA Observations of Aerosols above Clouds and their Interactions (ORACLES; https://espo.nasa.gov/ oracles) and Cloud-Aerosol-Radiation Interactions and Forcing (CLARIFY) field campaigns will soon provide in situ data that can be used to test these results and include the effects of aerosols during the African biomass burning season in the southeast Atlantic. 


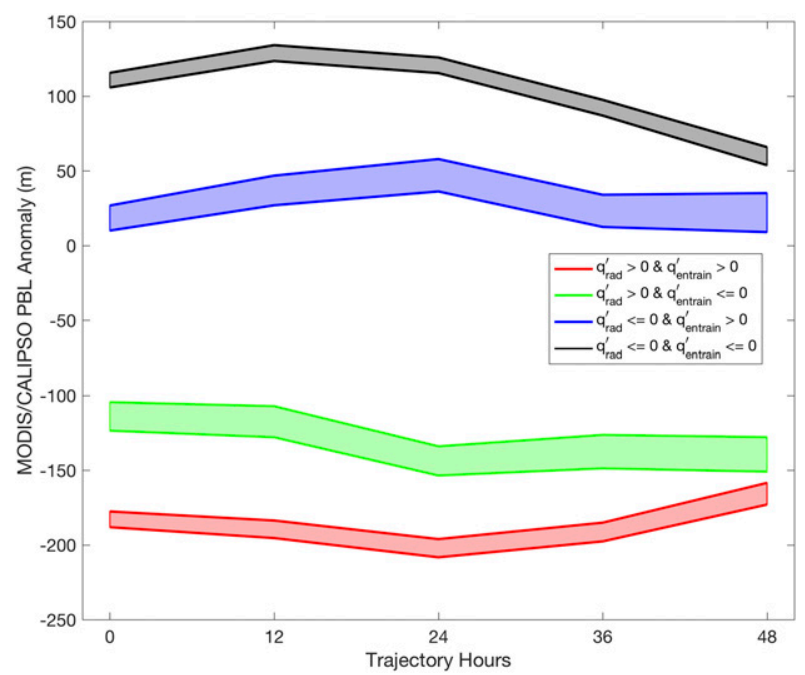

FIG. 12. The 48-h Lagrangian evolution of $\mathrm{PBL}^{\prime}$ for four sets of trajectories. The red line represents a set with above-mean radiating and entraining humidity. The green line represents a set with above-mean radiating humidity and below-mean entraining humidity. The blue line represents a group with below-mean radiating humidity and above-mean entraining humidity. The black line represents a group with below-mean radiating and entraining humidity. Humidity values are the mean averaged over the entire 48-h lifetime of each trajectory. The $2 \sigma$ standard error bounds are shown by the thickness of the lines.

The variable $\kappa$ was shown to be a reasonable but comparatively weak predictor of both PBL deepening and cloud cover evolution. This may be due to the ERAInterim dataset's inability to resolve the sharp contrasts in humidity and temperature across the inversion: $\kappa$ is calculated at two consecutive levels, but if the vertical resolution between levels is too coarse, then the signal may be reduced to some extent, which is likely given that there are only 12 pressure levels between 1000 and $700 \mathrm{hPa}$. In situ measurements of $\kappa$ may be better than those derived from coarse global reanalyses; $\kappa$ also lacks a radiative component, further reducing its effectiveness in predicting PBL deepening.

Results here show that subtropical Sc cloud decks may be equally sensitive to variations in overlying humidity as they are to variations in inversion strength, in contrast to Sandu et al. (2010) and Mauger and Norris (2010). In those works, it was shown that overlying humidity had a weaker, though still positive, relationship with Lagrangian cloud cover changes. Differences in sample size, statistical framework, and our ability to study one variable while holding the other constant may contribute to the discrepancy. Regardless, further study should be encouraged in both the observational and modeling realms.

Predicted increases in EIS (Qu et al. 2015) will likely be accompanied by changes in the humidity above the PBL. The cloud and boundary layer response to these

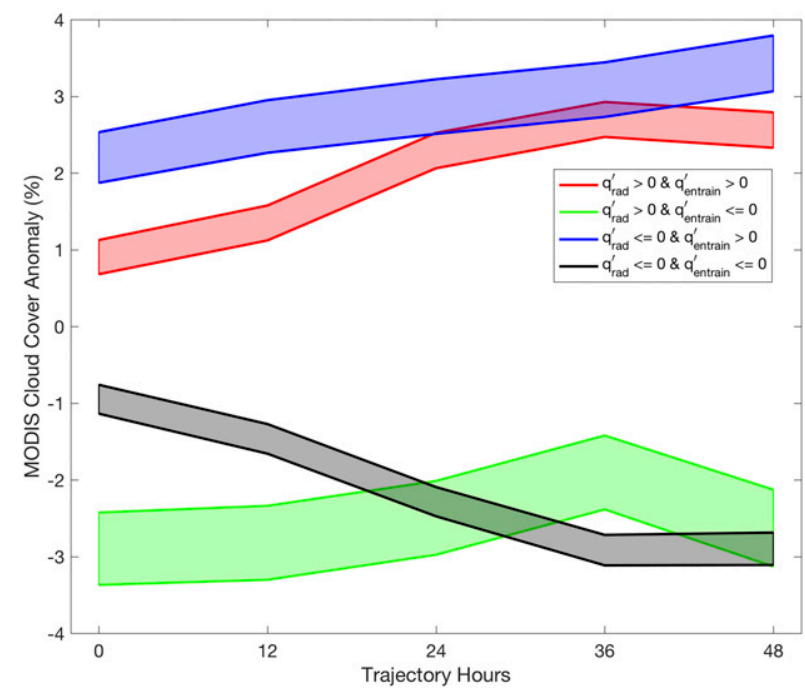

FIG. 13. As in Fig. 12, but for $\mathrm{CC}^{\prime}$.

changes will likely be sensitive to the distribution of humidity throughout the column. Future parameterizations describing the cloud response to tropospheric humidity will need to account for this sensitivity in order to properly quantify the cloud feedback to humidity changes. An increase in downwelling radiation accompanying more moisture entraining into the PBL could (all else being equal) lead to more cloud cover. However, humidity will be varying along with most other variables over the subtropical oceans, such as stability, subsidence, sea surface temperature, and wind speed, just to name a few. Therefore, in future work, the sign, strength, and location of any predicted humidity changes will not only need to be well predicted but also put into context with these other variables.

\section{Conclusions}

Here and in prior work, we show that inversion strength as defined by both LTS and EIS is anticorrelated with the specific humidity above the boundary layer, especially EIS. A strong inversion across the PBL and high humidity above the PBL both lead to greater cloud cover. The effect of the anticorrelation between stability and upper-level humidity is that the effects of these variables tend to obscure one another when tested as possible cloud- and PBL depth-controlling variables in the Lagrangian framework. The Lagrangian effects of LTS and EIS are only clearly evident when the humidity above the PBL is accounted for. After accounting for the confounding humidity, we show that strong inversions are indeed associated with increased cloud cover and decreased PBL depths on average when cloud scenes are tracked over $24 \mathrm{~h}$. 
Variables that account for the humidity and temperature gradient across the inversion, $\kappa$ and ECTEI, show some strengths and weaknesses as predictors of cloud and PBL evolution. The relationship between ECTEI and cloud cover evolution is the strongest of the variables tested, with a positive relationship observed. However, ECTEI was not a better predictor of PBL deepening than EIS. This may be due to the exclusion of the effects of longwave radiation emitted by the water vapor above the PBL; $\kappa$ shows weaker but sign-appropriate relationships with cloud cover evolution and PBL deepening. Increased $\kappa$ leads to an increase in cloud cover and a decrease in PBL depth. Issues with the $\kappa$ variable may stem from coarse reanalysis grid resolution, so this variable may be better suited to higher-resolution data.

We observe significant effects of humidity above the PBL on the Lagrangian changes in both cloud cover and PBL depths, with different mechanisms driving the effects. The humidity above the PBL was quantified in two ways: as the entraining humidity that enters the boundary layer and as the radiating humidity that sits above the entraining humidity and emits downwelling longwave radiation toward the PBL. The two quantities were tested independently in our Lagrangian framework. Results show that Lagrangian PBL deepening may be 3-4 times as sensitive to the radiative effects of the overlying humidity as the entraining effects, though both were shown to be significant. High humidity in a layer above the PBL was shown to suppress PBL deepening, while low humidity was associated with increased deepening. Lagrangian cloud cover evolution shows sensitivity to the entraining humidity but little to no sensitivity to the radiating humidity. The entrainment of increased water vapor into the boundary layer leads to more cloud cover.

Taken together, the above results highlight the need for the inclusion of the radiative effects of humidity when creating a quantity predicting the entrainment rate. These results also point to a cloud feedback response to climate warming and humidifying, with increased humidity possibly driving less of a decrease in subtropical stratocumulus clouds along a trajectory.

Acknowledgments. The authors thank Isabel McCoy and Hans Mohrmann for valuable collaboration. We thank Rhea George for trajectory software. This work was supported by NASA Grant NNXBAQ35G.

\section{REFERENCES}

Bretherton, C. S., P. N. Blossey, and C. R. Jones, 2013: Mechanisms of marine low cloud sensitivity to idealized climate perturbations: A single-LES exploration extending the CGILS cases. $J$. $A d v$ Model. Earth Syst., 5, 316-337, https://doi.org/10.1002/jame.20019.
Chen, T., W. B. Rossow, and Y. Zhang, 2000: Radiative effects of cloud-type variations. J. Climate, 13, 264-286, https://doi.org/ 10.1175/1520-0442(2000)013<0264:REOCTV>2.0.CO;2.

Christensen, M. W., G. G. Carrió, G. L. Stephens, and W. R. Cotton, 2013: Radiative impacts of free-tropospheric clouds on the properties of marine stratocumulus. J. Atmos. Sci., 70, 31023118, https://doi.org/10.1175/JAS-D-12-0287.1.

Deardorff, J. W., 1980: Cloud top entrainment instability. J. Atmos. Sci., 37, 131-147, https://doi.org/10.1175/1520-0469(1980)037<0131: CTEI $>2.0 . \mathrm{CO} ; 2$.

Dee, D. P., and Coauthors, 2011: The ERA-Interim reanalysis: Configuration and performance of the data assimilation system. Quart. J. Roy. Meteor. Soc., 137, 553-597, https://doi.org/ 10.1002/qj.828.

de Lozar, A., and J. P. Mellado, 2013: Direct numerical simulations of a smoke cloud-top mixing layer as a model for stratocumuli. J. Atmos. Sci., 70, 2356-2375, https://doi.org/10.1175/JAS-D-12-0333.1. , and - 2015: Mixing driven by radiative and evaporative cooling at the stratocumulus top. J. Atmos. Sci., 72, 4681-4700, https://doi.org/10.1175/JAS-D-15-0087.1.

Eastman, R., and R. Wood, 2016: Factors controlling low-cloud evolution over the eastern subtropical oceans: A Lagrangian perspective using the A-Train satellites. J. Atmos. Sci., 73, 331-351, https://doi.org/10.1175/JAS-D-15-0193.1.

, — , and C. S. Bretherton, 2016: Time scales of clouds and cloud-controlling variables in subtropical stratocumulus from a Lagrangian perspective. J. Atmos. Sci., 73, 3079-3091, https://doi.org/10.1175/JAS-D-16-0050.1.

$\longrightarrow,-$, and K. T. O, 2017: The subtropical stratocumulus-topped planetary boundary layer: A climatology and the Lagrangian evolution. J. Atmos. Sci., 74, 2633-2656, https://doi.org/10.1175/ JAS-D-16-0336.1.

Galewsky, J., 2018: Using stable isotopes in water vapor to diagnose relationships between lower-tropospheric stability, mixing, and low-cloud cover near the island of Hawaii. Geophys. Res. Lett., 45, 297-305, https://doi.org/10.1002/2017GL075770.

Greenwald, T. J., G. L. Stephens, S. A. Christopher, and T. H. Vonder Haar, 1995: Observations of the global characteristics and regional radiative effects of marine cloud liquid water. J. Climate, 8, 2928-2946, https://doi.org/10.1175/1520-0442(1995)008<2928: OOTGCA $>2.0 . \mathrm{CO} ; 2$

Hartmann, D. L., M. E. Ockert-Bell, and M. L. Michelsen, 1992: The effect of cloud type on Earth's energy balance: Global analysis. J. Climate, 5, 1281-1304, https://doi.org/10.1175/1520-0442(1992) 005<1281:TEOCTO $>2.0$. CO;2.

Henderson, D. S., T. L'Ecuyer, G. L. Stephens, P. Partain, and M. Sekiguchi, 2013: A multisensor perspective on the radiative impacts of clouds and aerosols. J. Appl. Meteor. Climatol., 52, 853-871, https://doi.org/10.1175/JAMC-D-12-025.1.

Hubanks, P. A., M. D. King, S. Platnick, and R. Pincus, 2008: MODIS atmosphere L3 gridded product algorithm theoretical basis document. MODIS Tech. Doc. ATBD-MOD-30, 96 pp.

Kawai, H., K. Tsuyoshi, and M. J. Webb, 2017: Interpretation of factors controlling low cloud cover and low cloud feedback using a unified predictive index. J. Climate, 30, 9119-9131, https://doi.org/10.1175/JCLI-D-16-0825.1.

King, M. D., and Coauthors, 2003: Cloud and aerosol properties, precipitable water, and profiles of temperature and water vapor from MODIS. IEEE Trans. Geosci. Remote Sens., 41, 442-458, https://doi.org/10.1109/TGRS.2002.808226.

Klein, S. A., and D. L. Hartmann, 1993: The seasonal cycle of low stratiform clouds. J. Climate, 6, 1587-1606, https://doi.org/ 10.1175/1520-0442(1993)006<1587:TSCOLS > 2.0.CO;2 
Krueger, S. K., G. T. McLean, and Q. Fu, 1995: Numerical simulation of the stratus-to-cumulus transition in the subtropical marine boundary layer. Part I: Boundary-layer structure. J. Atmos. Sci., 52, 2839-2850, https://doi.org/10.1175/1520-0469(1995)052<2839: NSOTST $>2.0 . \mathrm{CO} ; 2$.

Lawrence, M. G., 2005: The relationship between relative humidity and the dewpoint temperature in moist air: A simple conversion and applications. Bull. Amer. Meteor. Soc., 86, 225-233, https://doi.org/10.1175/BAMS-86-2-225.

Lilly, D. K., 1968: Models of cloud-topped mixed layers under a strong inversion. Quart. J. Roy. Meteor. Soc., 94, 292-309, https://doi.org/10.1002/qj.49709440106.

Lock, A. P., and M. K. Macvean, 1999a: The generation of turbulence and entrainment by buoyancy reversal. Quart. J. Roy. Meteor. Soc., 125, 1017-1038, https://doi.org/10.1002/qj.49712555513.

$\longrightarrow$, and — 1999b: The parametrization of entrainment driven by surface heating and cloud-top cooling. Quart. J. Roy. Meteor. Soc., 125, 271-299, https://doi.org/10.1002/qj.49712555315.

Maddux, B. C., S. A. Ackerman, and S. Platnick, 2010: Viewing geometry dependencies in MODIS cloud products. J. Atmos. Oceanic Technol., 27, 1519-1528, https://doi.org/10.1175/ 2010JTECHA1432.1.

Mauger, G. S., and J. R. Norris, 2010: Assessing the impact of meteorological history on subtropical cloud fraction. J. Climate, 23, 2926-2940, https://doi.org/10.1175/2010JCLI3272.1.

Mellado, J. P., 2017: Cloud-top entrainment in stratocumulus clouds. Annu. Rev. Fluid Mech., 49, 145-169, https://doi.org/ 10.1146/annurev-fluid-010816-060231.

- B. Stevens, H. Schmidt, and N. Peters, 2009: Buoyancy reversal in cloud-top mixing layers. Quart. J. Roy. Meteor. Soc., 135, 963-978, https://doi.org/10.1002/qj.417.

Moeng, C.-H., P. P. Sullivan, and B. Stevens, 1999: Including radiative effects in an entrainment rate formula for buoyancydriven PBLs. J. Atmos. Sci., 56, 1031-1049, https://doi.org/ 10.1175/1520-0469(1999)056<1031:IREIAE > 2.0.CO;2.

Nicholls, S., 1989: The structure of radiatively driven convection in stratocumulus. Quart. J. Roy. Meteor. Soc., 115, 487-511, https://doi.org/10.1002/qj.49711548704.

Oreopoulos, L., 2005: The impact of subsampling on MODIS level-3 statistics of cloud optical thickness and effective radius. IEEE Trans. Geosci. Remote Sens., 43, 366-373, https://doi.org/ 10.1109/TGRS.2004.841247.

Paluch, I. R., and D. H. Lenschow, 1991: Stratiform cloud formation in the marine boundary layer. J. Atmos. Sci., 48, 2141-2158, https:// doi.org/10.1175/1520-0469(1991)048<2141:SCFITM>2.0.CO;2.

Qu, X., A. Hall, S. A. Klein, and P. M. Caldwell, 2015: The strength of the tropical inversion and its response to climate change in
18 CMIP5 models. Climate Dyn., 45, 375-396, https://doi.org/ 10.1007/s00382-014-2441-9.

Randall, D. A., 1980: Conditional instability of the first kind upside-down. J. Atmos. Sci., 37, 125-130, https://doi.org/10.1175/ 1520-0469(1980)037<0125:CIOTFK >2.0.CO;2.

_ - and Coauthors, 2007: Climate models and their evaluation. Climate Change 2007: The Physical Science Basis, S. Solomon et al., Eds., Cambridge University Press, 589-662.

Sandu, I., B. Stevens, and R. Pincus, 2010: On the transitions in marine boundary layer cloudiness. Atmos. Chem. Phys., 10, 2377-2391, https://doi.org/10.5194/acp-10-2377-2010.

Siems, S. T., and C. S. Bretherton, 1992: A numerical investigation of cloud-top entrainment instability and related experiments. Quart. J. Roy. Meteor. Soc., 118, 787-818, https://doi.org/ 10.1002/qj.49711850702.

Stephens, G. L., and T. J. Greenwald, 1991:The Earth's radiation budget in relation to atmospheric hydrology: 2. Observations of cloud effects. J. Geophys. Res., 96, 15 325-15340, https:// doi.org/10.1029/91JD00972.

Stevens, D. E., and C. S. Bretherton, 1999: Effects of resolution on the simulation of stratocumulus entrainment. Quart. J. Roy. Meteor. Soc., 125, 425-439, https://doi.org/10.1002/ qj.49712555403.

Vaughan, M., S. Young, D. Winker, K. Powell, A. Omar, Z. Liu, Y. Hu, and C. Hostetler, 2004: Fully automated analysis of space-based lidar data: An overview of the CALIPSO retrieval algorithms and data products. Laser Radar Techniques for Atmospheric Sensing, U. N. Singh, Ed., International Society for Optical Engineering (SPIE Proceedings, Vol. 5575), 16-30.

Wentz, F. J., and T. Meissner, 2004: AMSR-E/Aqua L2B global swath ocean products derived from Wentz algorithm (L3 LWP), version 2. National Snow and Ice Data Center, accessed 1 June 2017, www.remss.com/missions/amsr.

Wood, R., 2012: Stratocumulus clouds. Mon. Wea. Rev., 140, 2373 2423, https://doi.org/10.1175/MWR-D-11-00121.1.

— tween stratiform low cloud cover and lower-tropospheric stability. J. Climate, 19, 6425-6432, https://doi.org/10.1175/ JCLI3988.1.

Wyant, M. C., C. S. Bretherton, H. A. Rand, and D. E. Stevens, 1997: Numerical simulations and a conceptual model of the stratocumulus to trade cumulus transition. J. Atmos. Sci., $\mathbf{5 4}$ 168-192, https://doi.org/10.1175/1520-0469(1997)054<0168: NSAACM $>2.0 . \mathrm{CO} ; 2$.

Yamaguchi, T., and D. A. Randall, 2012: Cooling of entrained parcels in a large-eddy simulation. J. Atmos. Sci., 69, 11181136, https://doi.org/10.1175/JAS-D-11-080.1. 